\title{
A atuação de um médico do Programa Mais Médicos para o Brasil e a mudança processo de trabalho da equipe de Estratégia de Saúde da Família.
}

\section{The actuation of a physician from more physicians to Brazil program and the change in the working process in Family Health Stategy team.}

\section{Actuación del médico del Programa Más Médico para Brasil y el proceso de cambio del trabajo del equipo de la Strategia de Salud Familiar.}

Iliane Rezer BERTÃO ${ }^{1}$

RESUMO: Trata-se do relato, sob a ótica da supervisão acadêmica, da atuação de um médico intercambista vinculado ao Programa Mais Médicos para o Brasil (PMMB) que, após ter identificado o idoso como o maior segmento populacional do município, aliado ao alto índice de envelhecimento da população residente, define seu projeto de intervenção na saúde do idoso. Os objetivos para uma intervenção de impacto na saúde da população idosa buscou atingir 100\% da população cadastrada e, para que a meta fosse atingida, o processo de trabalho da equipe foi avaliado e modificado. A equipe foi capacitada ao enfrentamento dos principais problemas do envelhecimento, políticas públicas foram instituídas e houve uma qualificação da saúde do idoso, à partir da adesão destes às consultas programadas. Organizou-se um novo enfoque às visitas domiciliares, na saúde bucal e melhor abordagem à hipertensão, principal problema de saúde do idoso na localidade. A caderneta do idoso começou a ser utilizada, estabeleceu-se um registro específico dos problemas de saúde na Unidade Municipal e, no decorrer desse processo, sentiu-se a necessidade de ter no município um órgão de representação dos idosos, sendo criado o Conselho Municipal do Idoso. As metas físicas propostas não foram, em parte, atingidas, mas a mudança do processo de trabalho foi, sem dúvida, o maior benefício para a população idosa. O processo de envelhecimento da população é contínuo e o trabalho iniciado deverá ter continuidade, o que permitirá a avaliação do impacto desse novo olhar da equipe de estratégia de saúde da família, à saúde dos idosos, de um pequeno município da região central do estado do Rio Grande do Sul.

Palavras-chave: mais médicos; supervisão; saúde, idoso.

1 Supervisora Programa Mais Médicos 
ABSTRACT: This is the report, under the academic supervision point of view, of the performance of an exchangee physician bounded to the Program More Doctors to Brazil (PMDB) that, after identified the elderly as the biggest populational segment of the city, allied to the high level of aging of the local population, defines his project of intervention in the elderly health. The established objectives, so that there was a significant intervention in the elderly population health, had the goal of achieving $100 \%$ of the registered population and, in order that the goal was reached, the teamwork process was valued and needed modifications. The modification happened, one team capable to face the aging main problems was constituted, public politics were instituted and there was an improvement in the aging of the elderly after the adherence of these in the programmed consultations, of the new focus in the domiciliary visits, of the mouth health attention and of the better approaching to the hypertension (the main problem in the polesine elderly population health). The "elderly booklet" began to be utilized, the elderly people began to have their specific health problems registered in the City Unity and, in the course of this project, the need of an elderly representation organ was felt, being created the City Council of the Elderly. The physical goals proposed were not fully achieved, but the work process modification was, undoubtedly, the biggest benefit to the elderly population. The process of the populational aging is continuous and the work that has been iniciated should be continued, which will allow the evaluation of the impact of this new focus of the family health strategy team in the elderly population of São João do Polesine's health.

Key words: more doctors; supervision, health, elderly

RESUMEN: Esta es la historia desde la perspectiva de la supervisión académica, del papel de un médico relacionado con el Programa Más Médicos para Brasil (PMMB) que, después de haber identificado a los ancianos como el grupo de población más grande de la ciudad, junto con la alta tasa de envejecimiento de la población residente, define su proyecto de intervención en la salud de las personas de la tercera edad. Los objetivos establecidos para que hubiese una intervención de impacto en la salud de la población anciana, tenían la meta de alcanzar el 100\% de la población registrada, para lograr la meta el proceso de trabajo del equipo fue evaluada y necesito cambios. El cambio ocurrió, un equipo capacitado para hacer frente a los principales problemas del envejecimiento se constituyo, se introdujeron políticas públicas y hubo una calificación de la salud de los ancianos, a partir de la adhesión de los mismos a las consultas programadas, el nuevo enfoque en las visitas a domicilio, cuidado de la salud bucal, el mejor enfoque para la hipertensión, el principal problema de salud de los polesiniense ancianos. El cuaderno de los ancianos comenzó a ser utilizado, las personas mayores tienen ahora registro específico de sus problemas de salud en la Unidad Municipal y, en el proceso, se sintió la necesidad de tener en el municipio un órgano que representara a las personas mayores, fue creado el Consejo Municipal de las personas mayores. Las metas físicas propuestas no fueron alcanzadas, en parte, pero el cambio en el proceso de trabajo fue, sin duda, el mayor beneficio para la población de edad avanzada. El envejecimiento de la población es continuo y el trabajo comenzado debe continuar, lo que permitirá evaluar el impacto de esta nueva visión del equipo de trabajo de salud de la familia, a la salud de las personas 
mayores en un municipio de la región central de Río Grande Sur.

Palabras clave: más médicos; supervisión; salud, tercera edad.

\section{INTRODUÇÃO}

O Programa Mais Médicos para o Brasil (PMMB), instituído pela Medida Provisória 621, em oito de julho de 2013, sancionado pela Lei 12871, de 22 de outubro de 2013, surge com o propósito de levar o atendimento médico às regiões prioritárias do SUS, incluindo os recantos mais distantes, onde notadamente existem os vazios assistenciais. ${ }^{1}$

Agregado a isso, o programa propõe-se a fortalecer a Atenção Básica, principal porta de entrada do Sistema Único de Saúde (SUS)², aprimorando a formação médica, proporcionando maior vivência prática e ampliando a inserção do profissional médico nas unidades de atendimento do SUS.

Para a garantia de um atendimento qualificado, o Programa visa intensificar a educação permanente, promovendo a integração entre ensino e serviço, através da atuação das instituições de ensino superior, na supervisão médico acadêmica, das atividades. Após dois anos da criação do $\mathrm{PMMB}$, o país começa a avaliar o impacto que causou no atendimento à população. ${ }^{1}$ Notadamente, foi oferecido a essas populações um atendimento médico humanizado, de qualidade monitorada ${ }^{3}$ permanentemente dando-lhes a dignidade de ter, mesmo que minimamente, seus problemas de saúde atendidos, quer pela atuação profissional, ou simplesmente, pela escuta qualificada, o que é feito, especialmente, na atuação do profissional na Estratégia de Saúde da Família (ESF), norteadora das ações de Atenção Primária à Saúde (APS)².

A cobertura da população atendida pela Estratégia de Saúde da Família, que alcançou um aumento de $135 \%$ desde 2002, teve a contribuição do PMMB, que beneficiou mais de 63 milhões de pessoas, segundo pesquisas divulgadas no site oficial do programa ${ }^{1}$. Isso permitiu uma maior atuação na prevenção e na promoção da saúde da população, e de forma mais eficaz, pois leva o profissional para junto da família,fazendo com que seja facilitada a observação das condições que predispõe os indivíduos ao adoecimento3.

No processo de implantação do PMMB nos municípios, o papel do médico supervisor4 contempla, além do suporte individual ao médico do programa, a observação do processo de trabalho da equipe na qual o médico está inserido, permitindo uma interação com as equipes municipais, o que pode suscitar reavaliação do processo.

Através da supervisão4, pode-se perceber que a consolidação do Programa, possibilitou aos municípios, uma avaliação aprimorada de seus principais problemas de saúde, o que, por si só, melhora o atendimento à população. Além disso, através do PMMB, o município pode conscientizarse do principal segmento de sua população e buscar políticas públicas de saúde, que possam 
qualificar seu atendimento, concretizando um salto de qualidade à saúde.

Foi o que ocorreu em São João do Polêsine, um município de colonização italiana, localizado na Região Central do Estado do Rio Grande do Sul que possui 2.635 habitantes, segundo o censo de 2010 do IBGE (Instituto Brasileiro de Geografia e Estatística), com 613 idosos, representando $23,26 \%$ da população total, sendo o $14^{\circ}$ município de maior população idosa do Brasil5. Além disso, o município tem o maior índice de envelhecimento da $4^{\text {a }}$ Coordenadoria Regional de Saúde, do Estado do Rio Grande do Sul5 e, até a chegada do médico do PMMB, não estava preparada para o envelhecimento populacional, não tendo, por isso, políticas públicas voltadas para esse segmento populacional. Diante dessa realidade, que também foi enfatizada num dos primeiros encontros loco-regionais de supervisão4, o médico traçou seu plano de metas para qualificar a saúde do idoso polesiniense.

Na escolha do Projeto de intervenção, do Curso de Especialização oferecido pela Universidade Federal de Pelotas, o médico optou pela Saúde do Idoso, inserindo assim, atividades que buscaram promover o atendimento ao idoso preconizado pela Política Nacional do Idoso, instituída pelo Ministério da Saúde em 2006, e que coloca a ESF como mobilizadora de ações que promovam uma saúde de qualidade à pessoa idosa6.

O objetivo desse relato é descrever a observação feita, através da atuação da supervisão acadêmica, das mudanças ocorridas na saúde do município de São João do Polêsine, com a chegada do médico intercambista e sua opção por Saúde do Idoso, como projeto de intervenção, em seu curso de especialização.

\section{DESENVOLVIMENTO}

Trata-se de um relato da atuação do médico, do PMMB, direcionada para atenção ao idoso, desde sua chegada no município, contemplando as ações propostas, para que seu projeto de intervenção tivesse êxito, sob a ótica da supervisão acadêmica4.

O Rio Grande do Sul é dividido de forma administrativa em 19 regiões de saúde. Santa Maria é a sede da $4^{\text {a }}$ regional e o Município de São João do Polêsine, com um índice de envelhecimento de 146, é um dos 32 municípios que compõe a regional7.

O Município possui uma unidade de saúde, que concentra secretaria municipal, farmácia municipal e atendimento médico e odontológico. Tem 100\% de cobertura de Estratégia de Saúde da Família, com Saúde Bucal. Conta uma estrutura hospitalar, com 16 leitos7. Ao chegar no município, em março de 2014, o médico José Patrício Herrera, formado pela Faculdade de Ciências Médica Faustino Pérez, de Cuba, em 1993, com RP:105254, teve seu período de adaptação, quando foi apresentado a equipe e buscou ter algum conhecimento sobre a situação de saúde da população.

Ao iniciar, efetivamente, suas atividades, deparou-se com um grande número de atendimentos, 
feitos por livre demanda, tendo permanecido, por um período como único médico trabalhando no município. A gestão municipal atenta a esse problema, contrata plantão permanente no hospital do município, para onde o intercambista pôde encaminhar as urgências, sendo a população orientada sobre esse fluxo de atendimento e permanecendo na Unidade de Saúde, apenas os casos manejados pela APS.

O transcorrer do atendimento das consultas, demonstra ao profissional expressiva quantidade de idosos atendidos na unidade de saúde. No terceiro mês da atuação da supervisão acadêmica, é promovida uma capacitação em saúde do idoso. Nesse evento a coordenação regional da saúde do idoso, da $4^{\text {a }}$ CRS, ao apresentar a Política de Saúde da Pessoa Idosa6, repassa aos médicos o índice de envelhecimento de cada uma das cidades que compõe a regional, sendo salientado São João do Polêsine, como o município de maior índice.

No referido evento, os médicos assistiram, ministrada pela supervisão, a palestra: "Os Cinco Gigantes da Geriatria." Esse conhecimento foi instigante e, aliado ao que já havia constatado pela vivência clínica diária na Unidade de Saúde, permitiu profissional a definição de um tema a ser utilizado para Projeto de Intervenção, proposto pelo curso de especialização. A escolha de um tema contemplando uma realidade de saúde, ainda manejada pelo município, determinou a necessidade do envolvimento de todos os atores da rede de atenção básica municipal e, o município de São João do Polêsine abraçou a oportunidade de qualificar o atendimento ao idoso residente.

A elaboração do plano de trabalho, para que o projeto acontecesse, passou pelo real cadastramento do número de idosos e pela avaliação de quais instrumentos seriam necessários para qualificar a atenção ao idoso. São elencados dois objetivos principais, a saber8:

1- Ampliar a cobertura do Programa de Saúde do Idoso8, tendo como meta expandir a atenção à saúde do idoso para $80 \%$ dos idosos;

2- Melhorar a qualidade da Atenção ao Idoso na Unidade de Saúde8, que teve dez metas propostas, todas abrangendo $100 \%$ dos idosos: avaliação multidimensional rápida; exame clínico apropriado ao idoso; exames complementares periódicos para hipertensos e diabéticos identificados; priorizar a prescrição de medicamentos da Farmácia Popular; cadastrar 100\% dos idosos acamados ou com problemas de locomoção; visitas domiciliares em 100\% dos idosos acamados ou com problemas de locomoção; rastreamento para Hipertensão; rastreamento dos idosos com Pressão Arterial sustentada acima de 130/80mmhg com diagnóstico já estabelecido de Hipertensão ou Diabete; avaliação da necessidade de atendimento odontológico; primeira consulta odontológica;

3 - Melhorar a adesão do Idoso ao Programa de Saúde do Idoso, com a meta de buscar 100\% dos faltosos às consultas programadas;

4 - Melhorar o Registro das Informações, com duas metas propostas: manter registro específico do atendimento dos idosos e distribuir Caderneta do Idoso para 100\% dos idosos. Para que os 
objetivos propostos fossem alcançados, alguns pontos necessitaram atenção e ajustes, como: o cadastramento de $100 \%$ dos idosos; a capacitação dos profissionais da equipe; a abordagem diferenciada das visitas domiciliares e do acolhimento ao idoso na unidade de saúde; a identificação do principal problema de saúde desta população; o agendamento de consultas, qualificação do profissional médico nas principais alterações do processo de envelhecimento, buscando, através da literatura, protocolos que contemplem o atendimento de rotina ao idoso saudável e ao idoso senil, em especial ao hipertenso; e a procura por exemplares da Caderneta do Idoso.

O cadastramento encontrou 767 idosos residentes em São João do Polêsine, sendo que no censo IBGE - 2010 foram identificados 613 idosos8. A capacitação da equipe deu-se pela interação desta, com a equipe de Saúde do Idoso da coordenadoria regional de saúde, da busca individual dos membros da equipe, por conhecimento técnico e seu posterior compartilhamento, e da assessoria prestada pelo PMMB, quer pela orientação do curso de especialização, quer pela supervisão acadêmica.

O acolhimento diferenciado ao idoso na unidade, bem como a ampliação do foco das visitas domiciliares, permitindo a identificação de situações que provocam o adoecimento destes indivíduos, no seu contexto familiar ${ }^{3}$, foi possível através do conhecimento do Caderno número 19, da Atenção Básica - Envelhecimento e Saúde da Pessoa Idosa, do Ministério da Saúde9, por leitura individual dos membros da equipe, e pela apresentação deste Caderno, em reunião microrregional organizada pela supervisão dos médicos do PMMB, com a presença da Coordenação da Saúde do idoso, da $4^{\mathrm{a}}$ Coordenadoria Regional de Saúde que focou a capacitação em "Acolhimento"6.

Nesta reunião, houve ainda capacitação sobre "Conselho Municipal do Idoso" e uma palestra com o título: "Principais Demências no Idoso". Através das consultas médicas, da atuação qualificada da equipe, e de sua atuação na comunidade, foi possível o atendimento de 414 idosos, perfazendo $55.4 \%$ do total de idosos, abaixo dos $80 \%$ propostos, o que foi explicado pelo tempo de intervenção inferior ao projetado.

Houve ainda a identificação do principal problema de saúde dos idosos de Polêsine, que é a hipertensão, sendo que $69 \%$ dos idosos avaliados são hipertensos, o que está de acordo com os números da Sociedade Brasileira de Cardiologia, que diz que mais de 50\% dos idosos do Brasil, são acometidos pela doença. É identificado que alguns destes pacientes, mesmo com prescrição otimizada, mantém níveis pressóricos acima do esperado, e dois fatores são determinantes dessa condição: a não utilização da medicação de maneira adequada, aliada a falta de orientação para modificação do estilo de vida. A estratégia para enfrentar esse problema é contemplada pelo agendamento de consultas para os idosos, que até então, eram atendidos por demanda espontânea e necessitavam deslocar-se com antecipação à unidade para garantia de atendimento, o que levava a um acompanhamento inadequado da evolução da doença, sem atuação que permitisse evitar desfechos. 
O estilo de consulta dos médicos provenientes de Cuba, que subjetivamente foi avaliado como humanizado, comprovou-se objetivamente com o atendimento do médico intercambista de Polêsine e, isso possibilitou a adesão do pacientes às consultas de rotina. Elencado pela equipe, como forma de permitir o compartilhamento do estado de saúde do idoso, pelos diversos segmentos que com ele interagem, a Caderneta do Idoso, instrumento oficial do Ministério da Saúde, que teve a primeira tiragem em 2008 e, no primeiro semestre de 2014, lançada sua mais nova versão, pela coordenação saúde da pessoa idosa (COSAPI), foi distribuída, e passou a ser usada por $65.4 \%$ dos idosos atendidos. Apesar de não fazer parte do Projeto de Intervenção do profissional médico, a equipe de saúde, no decorrer do trabalho com a população idosa, percebeu a necessidade de um órgão de representação desse segmento populacional e, por não ter claro o papel do Conselho Municipal do Idoso, neste contexto, solicitou à supervisão maiores esclarecimentos. Foi organizada uma capacitação, já citada anteriormente, que através da coordenação Regional da saúde do Idoso, promoveu esclarecimentos e sensibilização sobre a criação do Conselho. O Conselho Municipal do Idoso de São João do Polêsine foi criado, alavancando um trabalho permanente na busca de qualidade de vida a uma população que envelhece.

POSSIBILIDADES: o envelhecimento é contínuo e a população precisa estar permanentemente sendo cuidada, para tanto, o profissional médico, juntamente com a equipe já traçam estratégias pra que o trabalho continue. Percebem a importância de estabelecer parcerias, visando ao desenvolvimento do trabalho Intersetorial (escolas, clubes, igrejas, associações e outros). Apostam na organização de grupos de idosos para discussão e troca de experiências |relativas à sua saúde e de como melhorar a qualidade de vida mantendo-se participante e ativo em sua comunidade, na criação de Núcleos de Apoio à Atenção Básica, permitindo que outros profissionais vem a juntar-se a equipe permitindo uma maior e melhor atuação nas questões alimentação, motricidade, mobilidade, distúrbios do humor.

Além disso, por ter chamado a atenção da equipe o grande número de idosos que, ou vivem sozinhos, ou permanecem sozinhos por um período do dia, está sendo estudado, junto a gestão municipal, a possibilidade da criação do que é chamado "creche do idoso".

\section{CONCLUSÃO}

Um dos principais objetivos do Programa Mais Médicos para o Brasil (PMMB), que é o fortalecimento da atenção básica10, foi levado a termo no município de São do Polêsine. Houve uma mudança no processo de trabalho da equipe de saúde da família, permitindo que o principal segmento da população, o idoso, tenha um atendimento de qualidade. Porém, o processo contínuo de envelhecimento requer um trabalho constante da atenção primária à saúde, com educação permanente da equipe de Estratégia de Saúde da Família, que coloca - se como norteadora desse cuidado. O PMMB, no município em questão, tem 18 meses e conseguiu mudanças importantes sim, mas que deverão continuar e atingir $100 \%$ dos idosos residentes, sendo a supervisão acadêmica uma possibilidade de acompanhamento da continuidade desse trabalho, bem como da 
posterior avaliação do impacto das ações instituídas, na promoção de um envelhecimento ativo6 e na prevenção de desfechos clínicos, referentes à principal morbidade que acomete os idosos da população residente.

\section{REFERÊNCIAS BIBLIOGRAFICAS}

1. Site Oficial do Programa Mais Médicos Para o Brasil - Ministério da Saúde: www. masimedicos.gov.br. Disponível em http:/maismedicos.saude.gov.br. Acesso em: 22 Set. 2015.

2. Política Nacional de Atenção Básica, Estratégia de Saúde da Família. Portaria 648. Portaria GM/MS 648 de 28 de março de 2006.

3. Carvalho CJA; Assunção RC; Bocchi SCM. Percepção dos profissionais que atuam na estratégia de saúde da família, quanto a assistência prestada aos idosos: revisão integrativa da literatura. Physis Revista de saúde Coletiva. Rio de Janeiro, 2010; 20(4).

4. UNA-SUS (Universidade Aberta do SUS). Atividades de Supervisão Acadêmica. Disponível em: https://sistema.unasus.gov.br/webportfolio. Acesso em: 22, 24, 25 Set. 2015.

5. Instituto Brasileiro de Geografia e Estatística (IBGE), censo 2010. Disponível em: www. ibge.gov.br. Acesso em: 22 Set. 2015.

6. Política Nacional de Saúde da Pessoa Idosa (PNSPI), Portaria GM nº 2.528 de 19 de outubro de 2006.

7. DATASUS, Ministério da Saúde, 2015. Disponível em: www.datasus.gov.br. Acesso em: 21 Set. 2015.

8. Gonzales JPH. Qualificação da Saúde do idoso no PSF de São João do Polêsine. Trabalho Conclusão do Curso Especialização em Saúde da Família, Para Médicos do programa Mais Médicos- Pelotas - UFPEL, 2015.

9. Cadernos de Atenção Básica. Envelhecimento e Saúde da Pessoa Idosa, no 19. Ministério da Saúde, 2006.

10. Lei 12871, de 22 de outubro de 2013. Disponível em: http:/maismedicos.gov.br . Acesso em: 24 Set 2015.

Artigo apresentado em 05-09-15

Artigo aprovado em 20-11-15

Artigo publicado no sistema em 30-12-15 